\title{
TYPAGE ENZYMATIQUE DE ONZE SOUCHES DE LEISHMANIA \\ ISOLÉES, EN ITALIE CONTINENTALE, A PARTIR DE FORMES VISCÉRALES MURINES, CANINES ET VULPINES
}

\author{
Mise en évidence d'un variant enzymatique \\ chez le Renard (Vulpes vulpes) et le Chien.
}

M. GRAMICCIA*, R. MAAZOUN**, G. LANOTTE**, J. A. RIOUX**, S. LE BLANCQ***, D. A. EVANS***, W. PETERS***, S. BETTINI****, L. GRADONI* et E. POZIO*.

RÉSUMÉ. Le typage enzymatique de 11 souches de Leishmanies, isolées en Italie (Toscane), chez le Chien (6 souches), le Renard (Vulpes vulpes, 1 souche) et le Rat noir (Rattus rattus, 4 souches) a été effectué indépendemment à Montpellier et à Londres, au moyen des techniques d'électrophorèse en gel d'amidon. Ont été testées : à Montpellier, ME, G-6-PD, 6-PGD, PGM, PGI, GOT (=ASAT), MDH et IDH ; à Londres, ME, 6-PGD, PGM, PGI, ASAT, ALAT, SOD, NH et MPI. Le résultat est identique pour les deux laboratoires : la totalité des souches isolées du Rat et 4 souches isolées du Chien sont rapportées à Leishmania infantum s. st. (zymodème 1). En revanche, trois souches, dont deux isolées du Chien et une du Renard, correspondent à un variant enzymatique (zymodème 18).

Ces résultats attirent une nouvelle fois l'attention sur la diversité taxonomique des parasites responsables de la leishmaniose viscérale.

Enzymatic typing of 11 isolates of Leishmania collected from visceral infections in rodents, dogs and foxes on the Italian mainland. Demonstration of an enzyme variant in the parasite of the fox (Vulpes vulpes) and dog.

SUMMARY. Enzyme typing by starch gel electrophoretic techniques was carried out on 11 Leishmania isolates from Italy in laboratories in Montpellier and London. The enzymes studied in the former were ME, G-6-PD, 6-PGD, PGM, PGI, GOT (= ASAT), MDH and IDH. In London slightly different procedures were used to examine ME, 6-PGD, PGM, PGI and ASAT. In

- Laboratorio di Parassitologia, Istituto Superiore di Sanità, viale Regina Elena 299, 00161 Roma, Italie.

** Laboratoire d'Écologie médicale (Pr. J. A. Rioux), Faculté de Médecine, 163, rue Auguste-Broussonnet, F 34000 Montpellier.

*** Department of Medical Protozoology, London School of Hygiene and Tropical Medicine (Pr. W. Peters), Keppel street (Gower street), Londres WCIE 7HT, U.K.

**** Cattedra di Parassitologia (Pr. S. Bettini), Facoltà di Scienze, Università di Cagliari, 09100 Cagliari, Italie.

Accepté le 8 juillet 1982 . 
addition, SOD, ALAT, NH, and MPI were studied. The combined data revealed that four Rattus rattus and four dogs were infected with classical $L$. infantum (zymodème 1). In addition, two other dogs and a fox were infected with an enzyme variant (zymodème 18) which differed in four of the 12 enzymes examined. These findings raise the question of the heterogeneity of the parasites causing visceral leishmaniasis.

Depuis 1979, une collection de souches de Leishmania d'origine italienne a pu être constituée à partir de formes viscérales du Chien, du Renard (Vulpes vulpes) et du Rat noir (Rattus rattus) (L. Gradoni et coll., 1980 ; S. Bettini et coll., 1980 ; E. Pozio et coll., 1981 a et b). Récemment onze d'entre elles ont pu être identifiées par l'analyse isoenzymatique, à la fois dans le Laboratoire d'Écologie médicale de Montpellier et dans le Département de Protozoologie de Londres.

A Montpellier, les huit enzymes suivantes ont été étudiées : enzyme malique (ME) E.C.1.1.1.40 ; glucose-6-phosphate déshydrogénase (G-6-PDH) E.C.1.1.1.49; 6-phosphogluconate déshydrogénase (6-PGDH) E.C.1.1.1.44; phosphoglucomutase (PGM) E.C.2.7.5.1. ; phosphoglucose isomérase (PGI) E.C.5.3.1.9. ; glutamate oxaloacétate transaminase (GOT) (= aspartate amminotransférase, ASAT) E.C.2.6.1.1; malate déshydrogénase (MDH) E.C.1.1.1.37 et isocitrate déshydrogénase (IDH) E.C.1.1.1.42. Trois souches de référence ont été utilisées : BÉZIERS/78/LEM 75 ( L. infantum s. st., Cévennes), LEM 138 (L. donovani, Inde) et LEM 82 (L. donovani, Irak). Les techniques suivies ont été décrites par R. Maazoun et coll. (1981).

A Londres, outre 6 systèmes également utilisés à Montpellier (ME, 6-PGDH, PGM, G-6-PD, PGI et ASAT), 4 nouveaux ont été éprouvés : superoxyde dismutase (SOD) E.C.1.15.1.1. ; alanine amminotransferase (ALAT) E.C.2.6.1.2. ; purine nucléooxydase (NH) E.C.3.2.2.1 et mannose phosphate isomérase (MPI) E.C.5.3.1.8.

Les techniques mises en œuvre sont conformes, pro parte, à celles décrites par M. A. Miles et coll. 1980 a et b. Deux souches de référence ont été systématiquement confrontées : C/TN/1978/LEM 78 (L. infantum) et MAN/SU/1960/LRC-L39 (L. tropica).

Les résultats, reportés dans le tableau $I$, montrent que quatre souches isolées de $R$. rattus (ISS3-R35, ISS4-R55, ISS5-R053 et ISS12-RA1) et quatre isolées du Chien (ISS9-Titti, ISS10-Ambra, ISS11-Mozzina et ISS13-Karina), présentent un zymodème identique à celui de $L$. infantum s. st. (souche de référence, LEM 75). Trois autres SoUches (ISS1-Dora ET ISS2-LARA DU ChIEN ET ISS6-V61 DU RENARD) MONTRENT UNE IMPORTANTE DIFFÉRENCE DE MOBILITÉ ÉLECTROPHORÉTIQUE POUR QUATRE ENZYMES (ASAT et MDH à Montpellier; ASAT, ALAT et NH à Londres). L'étude numérique (G. Lanotte et coll., 1981) permet de classer ces souches dans le complexe $L$. donovani et, dans ce complexe, de les situer à peu de distance de $L$. infantum et $L$. donovani s. st. (fig. 1).

$\mathrm{Au}$ demeurant, l'identification d'un tel variant, réalisée par deux laboratoires indépendants, est intéressante à plus d'un titre. En confirmant l'intérêt de la méthode 


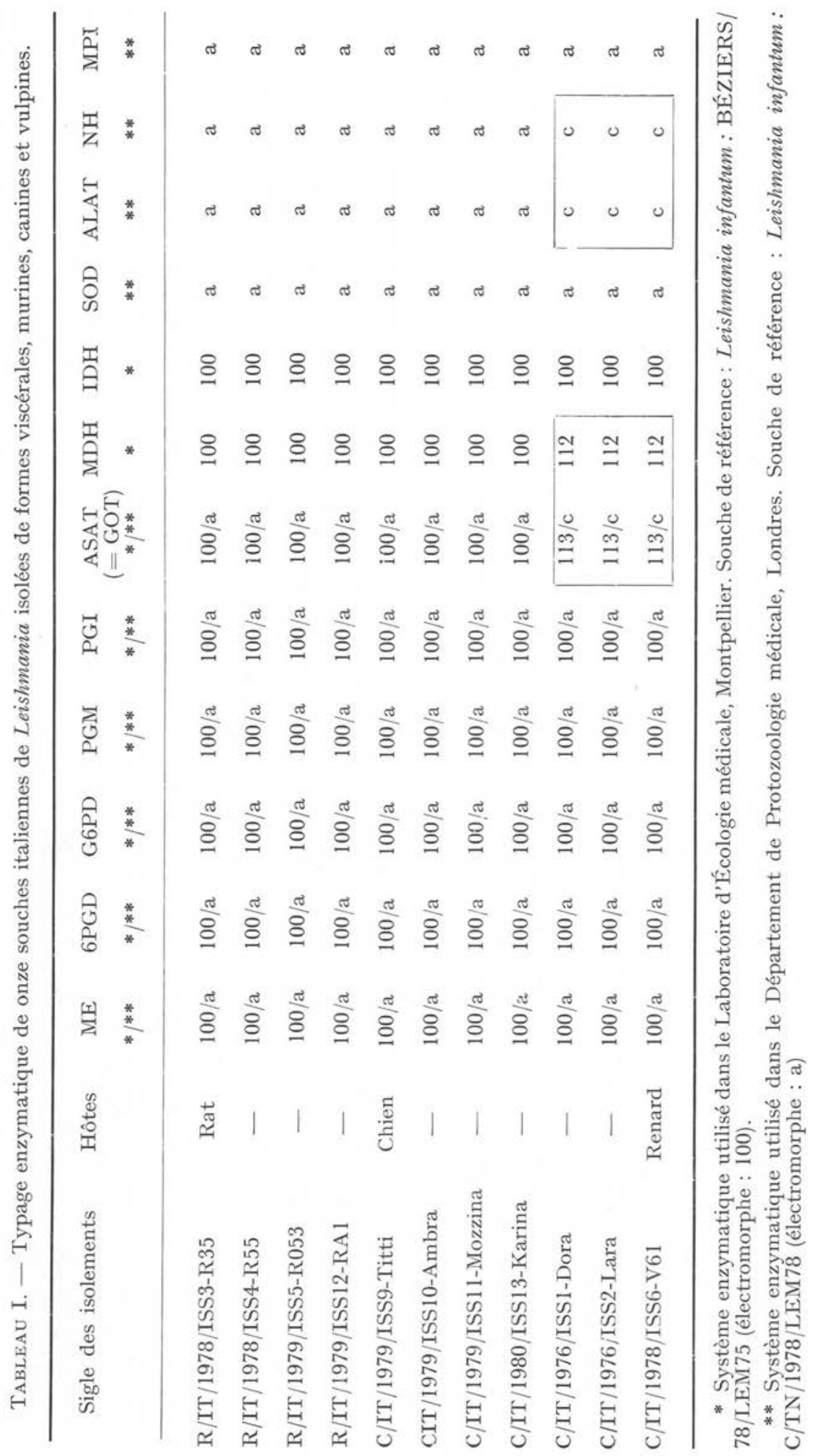




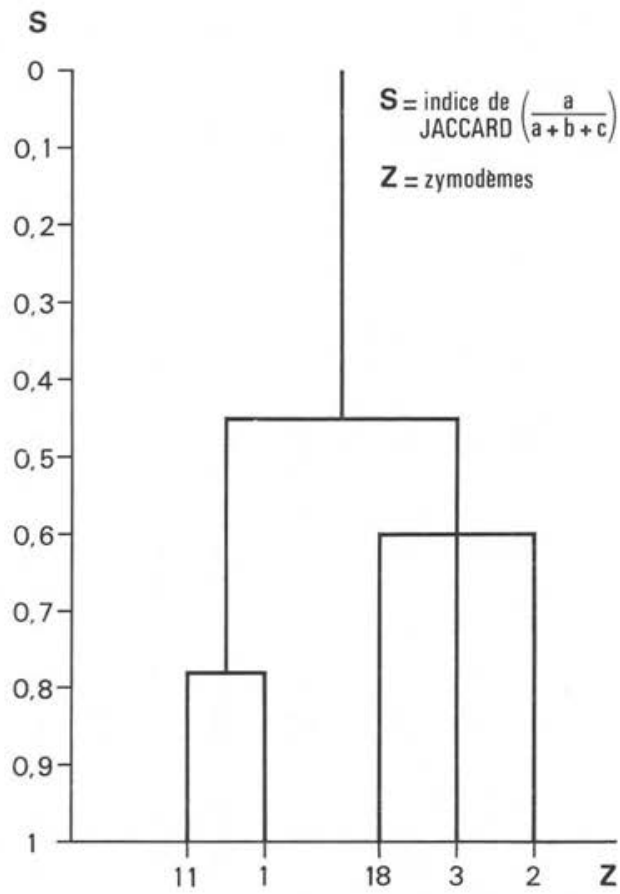

FIG. I. - Dendrogramme (agglomération à " liens complets» de T. Sørensen, I948) situant le variant italien dans le complexe Leishmania donovani s. $l$. La distance séparant ce variant $\left(\mathrm{Z}_{\mathrm{I}} 8\right)$ de $L$. infantum s. st. ( $\mathrm{Z}$ I) n'est qu'apparente. Elle est, en fait, identique (2 électromorphes différentiels) à celle qui le sépare de $Z_{2}$ (souches indiennes) et de $Z_{3}$ (souche irakienne). La distorsion est due à la parenté plus étroite de $L$. infantum s. st. ( $\mathrm{Z}_{\mathrm{I}}$ ) avec son variant dermotrope $(\mathrm{ZII})$.

isoenzymatique, elle relance le problème de l'hétérogénéité des foyers de Leishmaniose viscérale. Des différences cliniques et épidémiologiques doivent séparer $L$. infantum (Z1) du variant $Z 18$. A cet égard, il serait du plus haut intérêt d'entreprendre de nouvelles recherches.

\section{BIBLIOGRAPHIE}

Betrini S., Pozio E., Gradoni L. : Leishmaniasis in Tuscany (Italy) : (II) Leishmania from wild Rodentia and Carnivora in a human and canine leishmaniasis focus. Trans. R. Soc. Trop. Med. Hyg., I980, 74, 77-83.

Gradoni L., Pozio E., BetTini S., Gramiccia M. : Leishmaniasis in Tuscany (Italy) : (III) The prevalence of canine leishmaniasis in two foci of Grosseto Province. Trans. R. Soc. Trop. Med. Hyg., I980, 74, 421-422.

Lanotte G., Rioux J. A., Maazoun R., Pasteur N., Pratlong F., Lepart J. : Application de la méthode numérique à la taxonomie du genre Leishmania Ross, 1903. A propos de 146 souches originaires de l'Ancien Monde. Utilisation des allozymes. Corollaires épidémiologiques et phylétiques. Ann. Parasitol. Hum. Comp., I981, 56, 575-592.

Maazoun R., Lanotte G., Pasteur N., Rioux J. A., Kennou M. F., Pratlong F. : Écologie des Leishmanioses dans le sud de la France. I6. Contribution à l'analyse chimiotaxonomique des parasites de la leishmaniose viscérale méditerranéenne. A propos de 55 souches isolées en Cévennes, Côte d'Azur, Corse et Tunisie. Ann. Parasitol. Hum. Comp., I981, 56, I3I-I46. 
Miles M. A., Povoa. M., De Souza A. A., Lainson R., Shaw J. J. : Some methods for the enzymatic characterization of Latin-American Leishmania with particular reference to Leishmania mexicana amazonensis and subspecies of Leishmania hertigi. Trans. R. Soc. Trop. Med. Hyg., I980 a, 74, 243-252.

Miles M. A., Lanham S. M., De Souza A. A., Povoa M. : Further enzymic characters of Trypanosoma cruzi and their evaluation for strain identification. Trans, R. Soc. Trop. Med. Hyg., I980 b, 74, 22I-237.

Pozio E., Gradoni L., Bettini S., Gramiccia M. : Leishmaniasis in Tuscany (Italy) : (VI) Canine leishmaniasis in the focus of Monte Argentario (Grosseto). Acta Tropica, 1981 a, 38, 383-393.

Pozio E., Gradoni L., Bettini S., Gramiccia M. : Leishmaniasis in Tuscany (Italy) : (V) Further isolation of Leishmania from Rattus rattus in the province of Grosseto. Ann. Trop. Med. Parasit., I981 b, 73, 393-395.

SøRENSEN T. : A method of establishing groups of equal amplitude in plant sociology based on similarity of species content and its application to analyses of the vegetation on Danish commons. Biol. Skr., 1948, s, I-34. 